\title{
Research on Computerized Simulation of Occlusal Contacts for Complete Denture Based on 3D Digitized Modeling
}

\author{
Wei-can Chen \\ The First Department of Stomatology, Baoding No.1 Central Hospital,Baoding,China \\ 823322057@qq.com
}

\begin{abstract}
Keywords: Complete denture; Occlusal contacts; Laser scanning; 3D digitized model; Computerized simulation
\end{abstract}

\begin{abstract}
Aiming at solving the problems on dynamic occlusion of complete denture in the field of dental restoration, this paper presents a computerized system from the viewpoint of integration of related disciplines. Firstly the displacement matrix of mandibular movement on Hanau articulator is established by means of the method of the direction cosine matrix in spatial mechanism. Secondly by programming in Matlab 6.5 (a language for technical computing), VC6.0 ++ and VTK (Visualization ToolKit), and adopting these techniques such as laser scanning, computer graphics, and computer database, a 3D digitized model of the denture has been reconstructed; And then the 3D coordinate data of mark points are measured. On basis of this digitized model, the computerized simulation system of occlusal contacts for complete denture is developed. With the aid of this system, the dynamic occlusive procedure of the complete denture mounted on Hanau articulator is clearly observed at any required angle and location of views, in addition, the posit ions of the occlusal contacts is obtained. Therefore this study is helpful to research on the dynamic occlusion of complete denture and improve dental restorative procedures.
\end{abstract}

\section{Introduction}

In the field of oral and maxillofacial restoration, complete denture is an effective repair method for edentulous disease. After the denture produced, how to accurately detect the occlusal contact situation, make a better repair for edentulous patients by precision crafted denture, and achieve the uniform occlusal contact is the significant problem faced by denture repair clinicians[1]. For this purpose, this paper attempts to apply space agency theory, laser scanning 3D measurement, computer-aided engineering calculations, computer graphics, computer databases and other technology in the field of oral and maxillofacial restoration. Make a computer simulation and testing for complete denture of occlusal contacts, in order to aid clinical diagnosis doctor for complete denture work.

\section{The mandibular movement displacement matrix on Hanau articulator}

In order to achieve the simulation of occlusal contacts for complete denture, firstly, it needs to study the maxillary body movement on Hanau articulator (corresponding to natural tooth mandibular movement). Because the maxillary body movement on Hanau articulator is actually a coordinate transformation of coordinate system under the world coordinate system by following the movement of maxillary body. The small deformation of maxillary in the process of movement can be negligible, and consider it as a rigid body motion. Thus, a 3D cartesian coordinate system can be

established which is shown in Fig. 1. The first coordinate system is $O_{1 \times 01 \text { yolzo } 1}$, and the origin 
$O_{1}\left(x_{o 1}, y_{o 1}, z_{o 1}\right)$ is the geometric midpoint of the columns which is at the bottom of a horizontal plane of Hanua mandibular. The second coordinate system $O_{2 \text { хогуогzо2 }}$ is fixedly connected with the maxillary of Hanau articulator, and follows the movement of maxillary. The origin $O_{2}\left(x_{o 2}, y_{o 2}, z_{o 2}\right)$ is the geometric midpoint of the sliding condyle rod. The origin $O_{3}\left(x_{03}, y_{03}, z_{03}\right)$ of the third

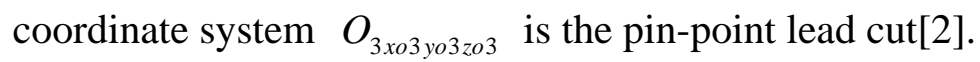

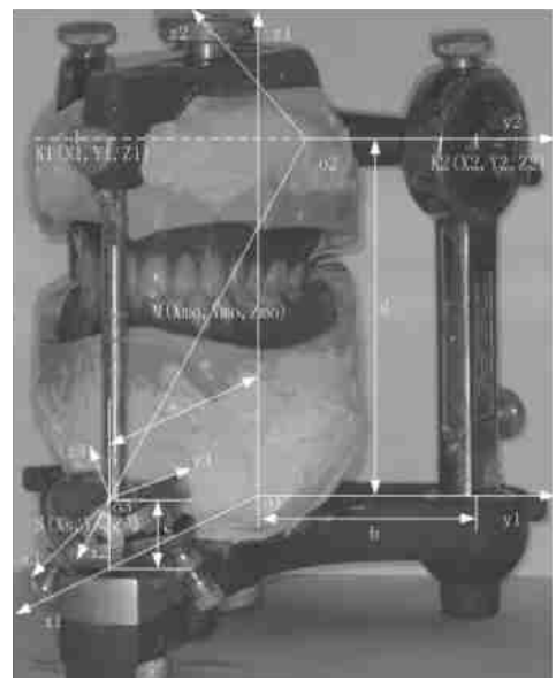

Fig. 1 Hanau articulator and the set of coordinate systems

Identification points are as follows (If not otherwise specified, the coordinates are expressed in the first coordinate system):

$K_{1}$---The axis of the left condyle ball, the coordinates is defined as $\left(x_{1}, y_{1}, z_{1}\right)$.

$K_{2}$---The axis of the right condyle ball, the coordinates is defined as $\left(x_{2}, y_{2}, z_{2}\right)$.

$S$---The point of incisal guidance pin, the coordinates is defined as $\left(x_{s}, y_{s}, z_{s}\right)$, and the coordinates in the third coordinates system is defined as $\left(x_{s r}, y_{s r}, z_{s r}\right)$.

$M$---Any point on the artificial maxillary surface, the coordinates is defined as $\left(x_{m}, y_{m}, z_{m}\right)$, and the coordinates on the jaw is defined as $\left(x_{m O}, y_{m o}, z_{m o}\right)$.

The fixed geometric parameters of Hanua articulator:

$b$--- The distance from $O_{1}$ to the central axis of jaw frame jamb. (mm)

$d$--- The distance from $O_{1}$ to the central axis of the sliding condyle rod on articulator. (mm)

The individual geometric parameters of Hanua articulator:

$a$--- The x-axis coordinate value of the incisal guidance pin $S$ on the jaw under the first coordinate system. (mm)

$c$--- The z-axis coordinate value of the incisal guidance pin $S$ on the jaw under the first coordinate system. (mm)

$\alpha$--- The angle between the tracks of incisal guidance pin moving on the incisal guidance surface and the axis of $\mathrm{O}_{3} y_{03}$.

According to the theory of space structure, the mandibular movement displacement matrix on Hanau articulator is established by applying the direction cosine matrix method. As shown in Fig. 2, set the maxillary point $P$ moves from the starting position $P_{1}$ to the end position $P_{2}$. The coordinate array of $P$ in $O_{1 x o 1 y o 1 z o 1}$ is defined as $(r)_{1}=\left(x_{1}, y_{1}, z_{1}\right)^{T}$. The coordinate array of $P$ in $\mathrm{O}_{2 \text { хо 2yozzo2 }}$ is defined as $(r)_{2}=\left(x_{2}, y_{2}, z_{2}\right)^{T}$. The coordinate array of origin $O_{2}$ in $O_{1 x o 1 y o 1 z o 1}$ is defined as $r_{1}^{\left(\mathrm{O}_{2}\right)}=\left(r_{1}^{\left(\mathrm{O}_{2}\right)}, r_{1}^{\left(\mathrm{O}_{2}\right)}, r_{1}^{\left(\mathrm{O}_{2}\right)}\right)^{t}$. The direction cosine matrix consistent with the coordinate system $O_{1 \times 1 y 1 z 1}$ through Rotation transformation of coordinate system $O_{2 \text { хог yo2zo2 }}$ is defined as $C_{12}$, thus, the following equation is established[3].

$$
(r)_{1}=\left(r_{1}^{\left(O_{2}\right)}\right)+\left[C_{12}\right](r)_{2}
$$


To further simplify the wording and calculation of matrix, we can introduce a so-called homogeneous coordinates $t=1$. Thus, the type of third-order matrix can be abbreviated to the following four order matrix form:

$$
\left(\begin{array}{c}
(r)_{1} \\
1
\end{array}\right)=\left[\begin{array}{cc}
{\left[C_{12}\right]} & \left(r_{1}^{\left(O_{2}\right)}\right) \\
0 & 1
\end{array}\right]\left(\begin{array}{c}
(r)_{2} \\
1
\end{array}\right)=\left[D_{12}\right]\left(\begin{array}{c}
(r)_{2} \\
1
\end{array}\right)
$$

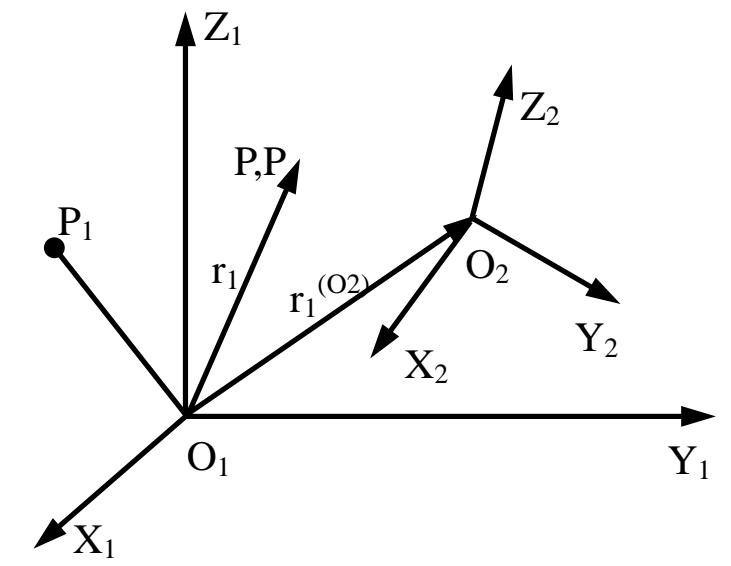

Fig. 2 The reference coordinate system for the development of displacement matrix

Since the displacement matrix $\left[D_{12}\right]$ is the four-order square, the solution of it should be based on the displacement arrays which belong to the movement status of any non-coplanar four points $A, B, C, D$ on the maxillary. This paper selects point $A$ as the the middle jaw median maxillary artificial teeth cut point, point $B$ as the guide pin-point cut (ie $S$ ), point $C$ as the midpoint between $K_{1}$ and $K_{2}$ (ie $O_{2}$ ). The displacement matrix of points $A, B$ and $C$ can be obtained through mandibular movement mathematical model. Therefore, from Eq. (1), there is:

$$
\left(\begin{array}{cccc}
x_{A 2} & x_{B 2} & x_{C 2} & x_{D 2} \\
y_{A 2} & y_{B 2} & y_{C 2} & y_{D 2} \\
z_{A 2} & z_{B 2} & z_{C 2} & z_{D 2} \\
1 & 1 & 1 & 1
\end{array}\right)=\left[D_{12}\right]\left(\begin{array}{cccc}
x_{A 1} & x_{B 1} & x_{C 1} & x_{D 1} \\
y_{A 1} & y_{B 1} & y_{C 1} & y_{D 1} \\
z_{A 1} & z_{B 1} & z_{C 1} & z_{D 1} \\
1 & 1 & 1 & 1
\end{array}\right)
$$

Whereby available:

$$
\left[D_{12}\right]=\left(\begin{array}{cccc}
x_{A 2} & x_{B 2} & x_{C 2} & x_{D 2} \\
y_{A 2} & y_{B 2} & y_{C 2} & y_{D 2} \\
z_{A 2} & z_{B 2} & z_{C 2} & z_{D 2} \\
1 & 1 & 1 & 1
\end{array}\right) \circ\left(\begin{array}{cccc}
x_{A 1} & x_{B 1} & x_{C 1} & x_{D 1} \\
y_{A 1} & y_{B 1} & y_{C 1} & y_{D 1} \\
z_{A 1} & z_{B 1} & z_{C 1} & z_{D 1} \\
1 & 1 & 1 & 1
\end{array}\right)^{-1}
$$

Because the spatial position of the maxillary body as long as can be determined by point $A, B$ and $C$, so when using inverse matrix calculation method to compute displacement matrix $\left[D_{12}\right]$, the fourth point $D$ should be determined by the above three point. This paper emulates point $D$ as follows: Regard $C A B$ on the maxillary as a plane, on its normal $C N, C$ point from any amount of length $h$ (can be 1 or 10 ) may have a fourth point $D$. Position coordinates of the point by point $\mathrm{C}$ can be written as follows:

$$
\begin{aligned}
& x_{D}=x_{C}=h \cos (x, C N) \\
& y_{D}=y_{C}=h \cos (y, C N) \\
& z_{D}=z_{C}=h \cos (z, C N)
\end{aligned}
$$

Here, the normal vector direction cosine $C B$ can be calculated: 


$$
\begin{aligned}
C N & =i \cos (x, C N)+j \cos (y, C N)+k \cos (z, C N) \\
& =\frac{1}{\sin (C A, C B)}\left[\begin{array}{ccc}
i & j & k \\
x_{A}-x_{c} & y_{A}-y_{c} & z_{A}-z_{c} \\
x_{B}-x_{c} & y_{B}-y_{c} & z_{B}-z_{c}
\end{array}\right]=\frac{N_{x}^{i}+N_{y}^{i}+N_{z}^{i}}{\sqrt{N_{x}^{2}+N_{y}^{2}+N_{z}^{2}}}
\end{aligned}
$$

Among them, the number of the direction of the normal vector $C B$ is:

$$
\begin{aligned}
& N_{x}=\left(y_{A}-y_{C}\right)\left(z_{B}-z_{C}\right)-\left(y_{B}-y_{C}\right)\left(z_{A}-z_{C}\right) \\
& N_{y}=\left(y_{A}-y_{C}\right)\left(x_{B}-x_{C}\right)-\left(y_{B}-y_{C}\right)\left(x_{A}-x_{C}\right) \\
& N_{z}=\left(x_{A}-x_{C}\right)\left(y_{B}-y_{C}\right)-\left(x_{B}-x_{C}\right)\left(y_{A}-y_{C}\right)
\end{aligned}
$$

The above results are substituted into the Eq. (2), then ,the mandibular movement displacement matrix on Hanau articulator $\left[D_{12}\right]$ can be obtained.

\section{The 3D digital modeling and measurement of artificial dentition}

The artificial dentition model is taken 3D scanned by KLS 171 non-contact laser scanner which is manufactured by French KREON. The laser beam diameter is $0.01 \mathrm{~mm}$, the wavelength is $670 \mathrm{~nm}$, the scanning speed is 20000 points. The point cloud data obtained after scanning is preprocessed by artificial dentition 3D laser scanning module of occlusal contact computer simulation software system. The module is very popular in recent years. The Visualization ToolKit (VTK) widely used in computer graphics, image processing and visualization fields is a development tool, which filters the point cloud data and removes the noise point of them. Then, the NURBS surfaces fitting method is used to reconstruct a 3D solid model of artificial dentition. Select landmark on the front, side and back of the 3D solid model. Through the measurement function provided by the proposed module, the 3D coordinate data can be obtained. To ensure accuracy and reduce errors, the coordinates of each marker points have been three measurements, and the results were averaged.

\section{The computerized simulation of occlusal contacts for complete denture}

Because of the efficient numerical functions and excellent access to the database, Matlab is used in this paper to complies software program. After the mandibular movement displacement matrix obtained, Database Toolbox is applied to establish a data connection, and the data is stored in Microsoft SQL sever 2000 database. Then, regard VC6.0 ++ and VTK as thedevelopment platform, ADO components as the database access, the simulation software system is finally developed.

Here is a simulation instance. Select edentulous patient with no obvious jaw position, bite, TMJ and other abnormal signs and symptoms, age 73, female. According to the traditional method of making dentures, the individual parameters of type Hanau $\mathrm{H} 2$ is set as follows: condylar inclination guide $\alpha_{\text {cond }}$ is $28^{\circ}$, cut guide slope $\alpha_{\text {inci }}$ is $10^{\circ}$, and cut guide skewed degree $\alpha_{\text {rinc }}$ is $15^{\circ}$. The geometric parameters of Hanua articulator is shown in Table. 1.

Table. 1 Geometric parameters of Hanua articulator

\begin{tabular}{|c|c|}
\hline Geometric parameter & Value \\
\hline$a$ & $108.2 \mathrm{~mm}$ \\
\hline$b$ & $57.1 \mathrm{~mm}$ \\
\hline$c$ & $17.8 \mathrm{~mm}$ \\
\hline$d$ & $76 \mathrm{~mm}$ \\
\hline$\alpha$ & $25^{\circ}$ \\
\hline
\end{tabular}

Shelf the finish denture and fixed each transfer parameters. Through the measurement of laser scanning, the cut-point coordinates of maxillary artificial teeth is $(90.5,0,60.2) \mathrm{mm}$. The above 
data is put in the complete denture occlusion simulation software system, and simulate it. Among them, Fig. 3 shows the front and side view of centric occlusion, and Fig. 4 shows the Front and side view of right lateral occlusion. Through using the proposed software system the process of the mandibular occlusal contacts Hanau mandibular jaw holder kinematics can be reappeared. Moreover, through the arbitrary rotation, zoom and other operations of the 3D model, the whole process of mandibular occlusal contact can be comprehensively and clearly viewed from any angle and position. According to the Boolean functions provided by the software operating system, through the upper and lower three-dimensional Boolean operations solid model to calculate the intersecting portion, that is able to achieve the upper and lower contact position and the area for testing.

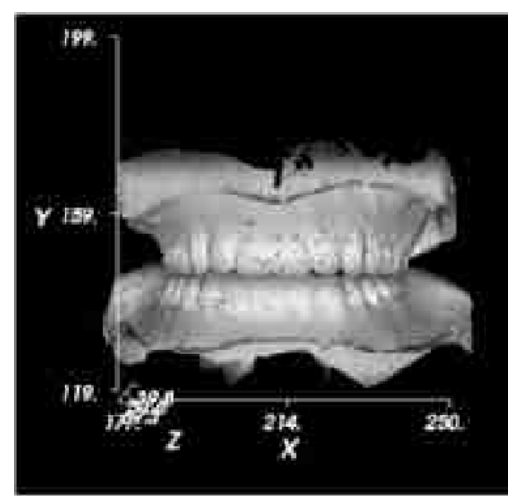

(a) Front

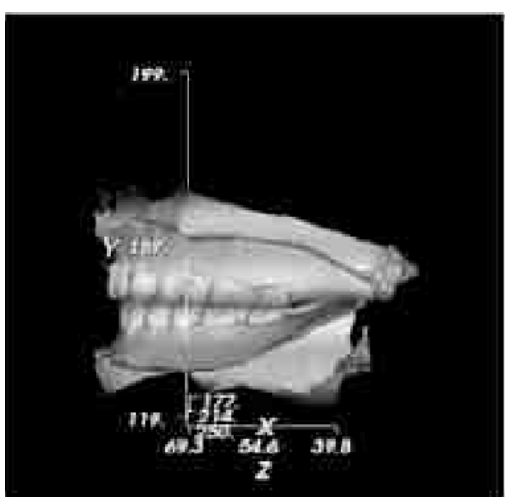

(2) Side

Fig. 3 The Front and side view of centric occlusion

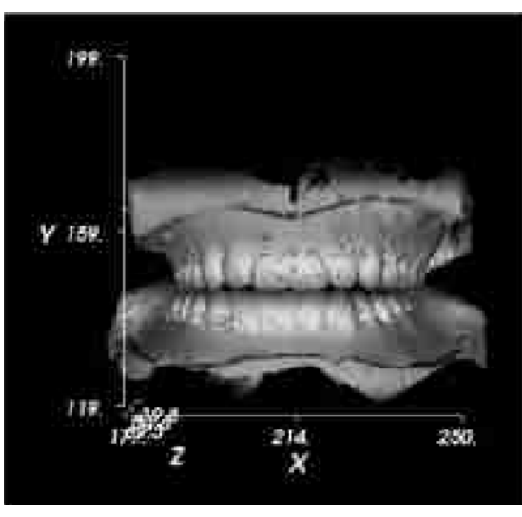

(a) Front

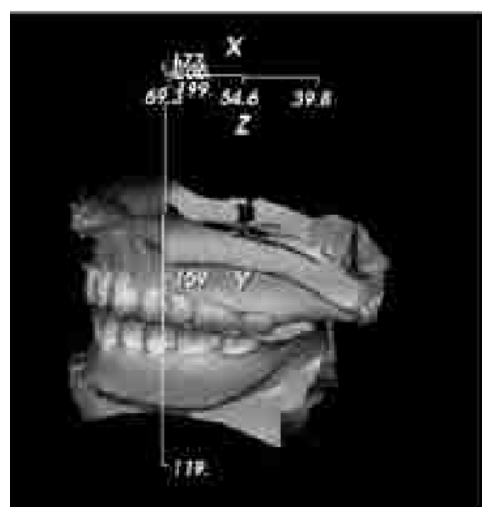

(2) Side

Fig. 4 The Front and side view of right lateral occlusion

\section{Conclusions}

This paper applies space agency theory, laser scanning 3D measurement, computer aided engineering calculations, computer graphics, computer databases and other technology in the field of oral and maxillofacial restoration. Make a computer simulation and testing for complete denture of occlusal contacts, in order to aid clinical diagnosis doctor for complete denture work. The whole process of mandibular occlusal contact can be comprehensively and clearly viewed from any angle and position by the proposed system, which lays a good foundation, and assists doctors to make a clinical diagnosis denture repair work.

\section{References}

[1] Lu Eryi, Zhang Fuqiang, Chen Xiaojun. Reliability of three-dimensional reconstruction and 
measurement system for complete denture occlusion. Journal of Shanghai Jiaotong University (Medical Science), Volume 28, Issue 2 (2008), P. 170-172.

[2] Chen Xiaojun, Lueryi, Chen Jianjun. Computerized simulation of occlusal contacts for complete denture based on 3D digitized modeling. Beijing Biomedical Engineering, Volume 25, Issue 1 (2006), P. 12-15.

[3] Lv Peijun, Wang Yong, Li Guozhen. Development of a system for robot aided teeth alignment of complete denture. Chin J Stomatol, Volume 36, Issue 2 (2001), P. 139-142. 